\title{
Significance of combined detection of JAK2V617F, MPL and CALR gene mutations in patients with essential thrombocythemia
}

\author{
LIYING JI, MENGYAO QIAN, NANA WU and JIANMIN WU \\ Institute of Genomic Medicine, Wenzhou Medical University, Wenzhou, Zhejiang 325000, P.R. China
}

Received March 16, 2016; Accepted November 3, 2016

DOI: $10.3892 /$ etm.2017.4077

\begin{abstract}
The aim of this study was to analyze the mutation rate of JAK2V617F, MPLW515L/K and CALR genes in adult patients with essential thrombocythemia (ET) and the accuracy of the combined detection by the receiver operating curve. Three hundred and forty-two cases with high-platelets $\left(\geq 300 \times 10^{9} / 1\right)$ were consecutively selected. The patients were analyzed for routine blood examination, bone marrow biopsy and genetic testing. One hundred and fifty-four cases (45.03\%) were diagnosed with ET and 188 cases of secondary thrombocythemia according to the hematopoietic and lymphoid tissue tumor classification standards of 2008. It was found that the mutant type of three genes showed three bands, whereas only one band for wild-type. The JAK2V617F and MPL mutations did not cause a change in the open reading frame and the CALR mutation resulted in its change. The mutation rate of JAK2V617F and CALR in ET group was significantly higher than that in the secondary thrombocythemia group $(\mathrm{p}<0.05)$. The positive mutation rate of MPL was only $4.55 \%$. JAK2V617F-positive mutation alone was used to diagnose with ET. The area under the curve (AUC) was 0.721 . The sensitivity was $72.4 \%$, the specificity was $79.5 \%$ and the cut-off value was 0.25 . When CALR-positive mutation alone was used to diagnose ET, the AUC, sensitivity, specificity and cut-off value were $0.664,68.4,82.4$ and $0.09 \%$, respectively. JAK2V617F combined with CALR mutation were used for diagnosis of ET. The AUC was 0.862 , the sensitivity was $85.9 \%$, the specificity was $87.8 \%$, and the cut-off values were 0.21 and 0.07 . In conclusion, the positive mutation rate of JAK2V617F and CALR in ET was higher, and the sensitivity, specificity and accuracy of the diagnosis of ET were significantly improved using the detection of JAK2V617F and CALR.
\end{abstract}

Correspondence to: $\mathrm{Dr}$ Jianmin $\mathrm{Wu}$, Institute of Genomic Medicine, Wenzhou Medical University, 268 Xueyuan Road, Wenzhou, Zhejiang 325000, P.R. China

E-mail: jianminwu81@tom.com

Key words: primary thrombocytopenia, JAK2V617F, MPLW515L/K, CALR, gene mutation, receiver operating curve

\section{Introduction}

Essential thrombocy themia (ET) is the most common subtype of classic Philadelphia chromosome negative bone marrow proliferation tumor (MPN). Bone marrow fibrosis, thrombus and risk of bleeding are the most common complications of ET. Approximately $10 \%$ of patients have acute leukemia transformation (1). In recent years, the ET rate has been on the increase annually, and approximately 5-8/10 million individuals were diagnosed with ET in 2005 (2).

ET may be the result of combined effects of gene and environment. JAK2V617F mutations can occur in $50-60 \%$ of ET patients, and the mutation-positive patients have a higher risk of thromboembolism. Reverse positive mutation status was negatively correlated with survival outcomes and serious adverse event occurrence, suggesting that the JAK2V617F mutation plays an important role in the pathogenesis of ET $(3,4)$. However, $50 \%$ of patients show a JAK2V617F-negative state. Recently, gene sequencing identified that MPLW515L/K and CALR gene of mutations can compensate for the deficiency of JAK2V617F negativity, which can provide an important basis for explaining genetic pathogenesis mechanism of ET $(5,6)$.

The aim of the present study was to analyze the mutation rate of JAK2V617F, MPLW515L/K and CALR genes in adult patients with essential thrombocythemia (ET) and the accuracy of the combined detection by the receiver operating curve.

\section{Patients and methods}

Patients. A total of 342 patients were selected consecutively in the Second Affiliated Hospital of Wenzhou Medical University with simple high platelet ( $\left.\geq 300 \times 10^{9} / 1\right)$ from January 2013 to January 2016. The platelet count was continuously monitored on weekly basis to take mean value. Exclusion criteria were: Patients with a high or low level of white blood cells, red blood cells or hemoglobin, history of recent surgery, bleeding, blood transfusion, pregnancy, malignant tumor, blood diseases such as leukemia and myeloproliferative disorders, severe hepatic and renal dysfunction, or those combined with nerve or spirit system diseases. Furthermore, patients with poor compliance, those who could not undergo surgery following various examinations, e.g., bone marrow biopsy and genetic testing, and those with incomplete data were excluded. All the patients were tested for detection of blood, bone marrow biopsy and 
the genes. The study was approved by the Ethics Committee of the Wenzhou Medical University. Patients or their family members provided written informed consent.

According to the hematopoietic and lymphoid tissue tumor on ET classification standards, 2008 (7): i) platelet count $\geq 450 \times 10^{9} / 1$; ii) bone marrow biopsy showed that mature and volume increased megakaryocyte increase dominated, with no obvious increase of neutrophils or polycythemia; iii) excluded were those with other myeloproliferative disorders, such as polycythemia vera, primary myelofibrosis and chronic myeloid leukemia; and iv) acquired JAK2V617F mutation or other clonal marker, or secondary thrombocythemia with no clonal markers. One hundred and fifty-four cases were diagnosed with ET $(45.03 \%)$ and 188 patients were diagnosed with secondary thrombocythemia. In the ET group, 86 patients were male and 68 female, aged 38-69 years and median age of 53.4 years and platelet of $463-3,547 \times 10^{9} / 1$ with average of $865.3 \pm 65.4 \times 10^{9} / 1$. Ninety-eight cases were male and 90 cases were female in the secondary thrombocythemia group, aged 34-72 years and median age of 56.3 years, platelets of $357-2,451 \times 10^{9} / 1$ and average of $73.28 \pm 82.3 \times 10^{9} / 1$. There were no significant differences in the gender, age, and platelet count between the two groups $(\mathrm{p}>0.05)$.

Gene detection method. Mutations in JAK2V617F and MPLW515L/K gene were detected by allele-specific polymerase chain reaction (AS-PCR). Reagents and equipment used were DNA extraction kit (Dynal, Oslo, Norway), PTC-200 ${ }^{\mathrm{TM}}$ PCR instrument (MJ Research, Inc., Waltham, MA, USA), CS-6R centrifuge (Beckman Coulter, Inc., Brea, CA, USA), the gel imaging analysis system (Transilluminator 202D; Cold Spring Harbor Laboratory, Cold Spring Harbor, NY, USA).

The main steps are as follows: i) DNA extraction: a) $500 \mu 1$ peripheral blood was extracted in a 2-ml EP tube. One milliliter of red blood cell lysis was added at $4^{\circ} \mathrm{C}$ and centrifuged at $8,000 \mathrm{x} \mathrm{g}$ for $1 \mathrm{~min}$; b) the supernatant was discarded and residual white lump in the pipe bottom was retained. Protease $(125 \mu \mathrm{l})$ was added with gentle mixing for $5 \mathrm{sec}$. Subsequently, the tube was placed in $65^{\circ} \mathrm{C}$ water bath for $10 \mathrm{~min}$ and gently mixed every 2 min until the precipitation was completely dissolved; iii) $275 \mu \mathrm{l}$ of protein clear solution was added with gentle mixing for $5 \mathrm{sec}$. It was followed by incubation at $-20^{\circ} \mathrm{C}$ for about $10 \mathrm{~min}$ and then centrifuged at $10,000 \mathrm{x} \mathrm{g}$ for $5 \mathrm{~min}$; d) the supernatant was discarded, and $1 \mathrm{ml}$ of $70 \%$ ethanol was added with gentle mixing for $5 \mathrm{sec}$. The tube contents were then centrifuged at $10,000 \mathrm{x} \mathrm{g}$ for $1 \mathrm{~min}$; e) the ethanol was discarded, and the DNA containing rube was placed at room temperature for $2 \mathrm{~min}$ for volatilization of extra alcohol. Subsequently, $100 \mu \mathrm{l}$ DNA dissolving solution was added and then tube was incubated at $65^{\circ} \mathrm{C}$ for 15 min to fully dissolve DNA; and f) DNA concentration and purity were measured with UV spectrophotometer.

ii) Primer sequences were designed for two forward primers and a reverse universal primer. PCR product was $203 \mathrm{bp}$ and the reference was $364 \mathrm{bp}$. Primers were produced by Shanghai Biological Engineering Co., Ltd. (Shanghai, China). Forward specific, 5'-AGCATTTGGTTTTAAATTATGGAGTAT ATT-3'; forward internal, 5'-ATCTATAGTCATGCTGAAA GTAGGAGAAAG-3' and reverse, 5'-CTGAATAGTCCTACA GTGTTTTCAGTTTCA-3'. iii) PCR reaction: $25 \mathrm{ng}$ of DNA (10 $\mathrm{mmol} / \mathrm{l}), 0.2$ of forward primer and $0.4 \mu \mathrm{l}(10 \mathrm{mmol} / \mathrm{l})$ reverse primer, 12.5 Master mix and double ionized water were added for final volume of $25 \mu \mathrm{l}$. PCR amplification cycle parameters were pre-denaturation at $95^{\circ} \mathrm{C}$ for $4 \mathrm{~min}$, denaturation at $95^{\circ} \mathrm{C}$ for $30 \mathrm{sec}$, annealing at $55^{\circ} \mathrm{C}$ for $30 \mathrm{sec}$, extension at $72^{\circ} \mathrm{C}$ for $1 \mathrm{~min}, 35$ cycles, final extension at $72^{\circ} \mathrm{C}$ for $7 \mathrm{~min} .1 .5 \%$ agarose gel containing ethidium bromide was prepared for the analysis. Five microliters of PCR products and $1 \mathrm{X}$ TBE buffer was mixed and added for electrophoresis at $90 \mathrm{~V}$ for $40 \mathrm{~min}$. The image was taken and analyzed with UV perspective image instrument.

iv) Gene sequencing: positive specimens were retrieved and purified after PCR amplification with the outside primers. Product was sent to Shanghai Yingjun Biotechnology Co. Ltd. (Shanghai, China) for sequencing. The result underwent alignment with the wild-type gene sequence. Semi-quantitative analysis results were shown as target gene copy number/corresponding wild-type gene copy number. MPLW515 primer sequences: forward primer M1, 5'-AGTAGGGGCTGGCTG GAT-3' (409 bp) and reverse M2, 5'-CTAGTCGCCGAGGTG AGC-3' (409 bp); specific primer M3, 5'-CCTGCTGCTGC TGAGGTTGC-3' (279 bp) and reverse M2, 5'-CTAGTCGCC GAGGTGAGC-3' (279 bp).

PCR reaction mixture consists of DNA $25 \mathrm{ng}, 12.5 \mu \mathrm{l}$ of 2X Taq PCR Master mix [KT201; Tiangen Biotech (Beijing) Co., Ltd., Beijing, China], $0.25 \mu 1$ for each M1, M3 primers (10 mmol/l), $0.5 \mu \mathrm{l}$ of $\mathrm{M} 2$ primer $(10 \mathrm{mmol} / \mathrm{l})$ and deionized water to make up final volume of $25 \mu \mathrm{l}$. PCR parameters and gene sequencing were similar to those above.

CALR gene mutation was detected by direct sequencing method. QIAamp DNA Mini kit (Qiagen, Valencia, CA, USA), exon 9 primers (Invitrogen, Carlsbad, CA, USA) of $5.0 \mathrm{pmol} / \mu \mathrm{l}$.

Primer sequence was: forward, 5'-CTGGTCCTGGTCCT GATGT-3' and reverse, 5'-TCTCACAGAGACATTATTTGGC.

PCR reaction mixture consists of $50 \mathrm{ng}$ DNA, $15 \mu 12 \mathrm{X}$ Taq PCR Master mix [KT201; Tiangen Biotech (Beijing) Co., Ltd.], $0.5 \mu \mathrm{l}$ for each primer and deionized water was added to make up final volume of $30 \mu \mathrm{l}$. PCR conditions were $98^{\circ} \mathrm{C}$ for $3 \mathrm{~min}$, $98^{\circ} \mathrm{C}$ for $10 \mathrm{sec}, 63^{\circ} \mathrm{C}$ for $30 \mathrm{sec}, 72^{\circ} \mathrm{C}$ for $30 \mathrm{sec}$ for $29 \mathrm{cycles}$, and final extension at $72^{\circ} \mathrm{C}$ for $5 \mathrm{~min}$. Gene sequencing was the same as above.

Observation index. The expression of JAK2V617F, MPLW515L/K and CALR gene positive mutation rate in two groups and the relative expression levels were measured.

Statistical analysis. SPSS 19.0 statistical software (Chicago, IL, USA) was used for data processing. Measurement data are presented as mean \pm standard deviation, the comparison between groups is shown using t-test. Countable data are presented as percentage, comparison between the groups were with $\chi^{2}$ test. The diagnostic sensitivity, specificity and accuracy was analyzed using the receiver operating curve (ROC). $\mathrm{P}<0.05$ indicates a statistically significant difference.

\section{Results}

Sequencing results of three gene mutations. The mutant type of the three genes showed two electrophoretic bands and the 

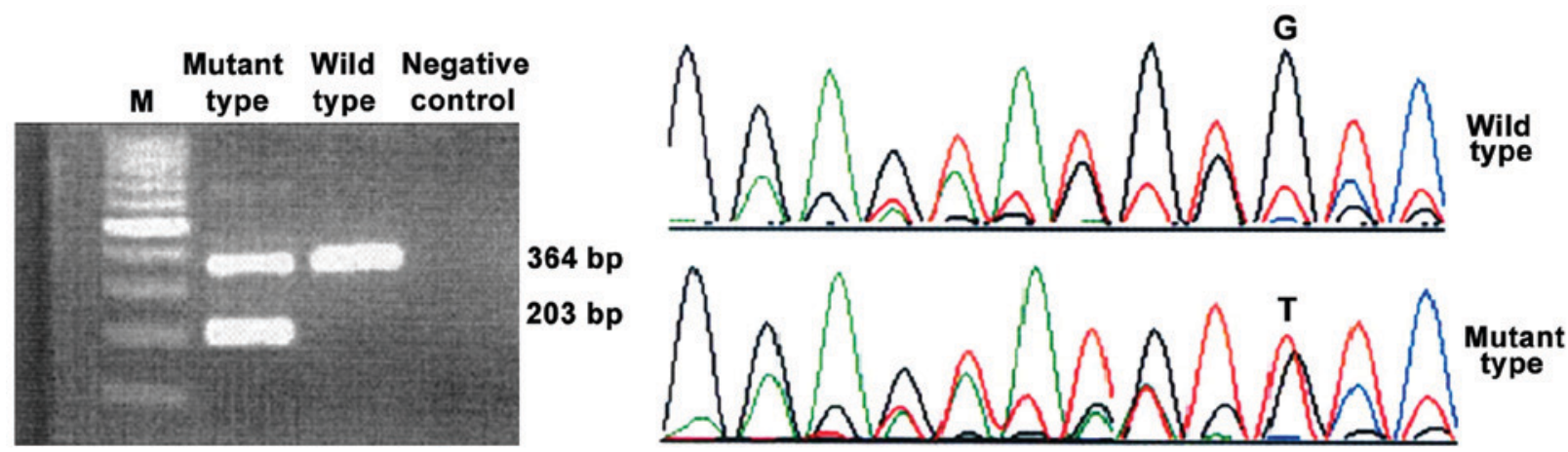

Figure 1. PCR and sequencing results of the JAK2V617F mutation (the black G was replaced by red T). PCR, polymerase chain reaction.
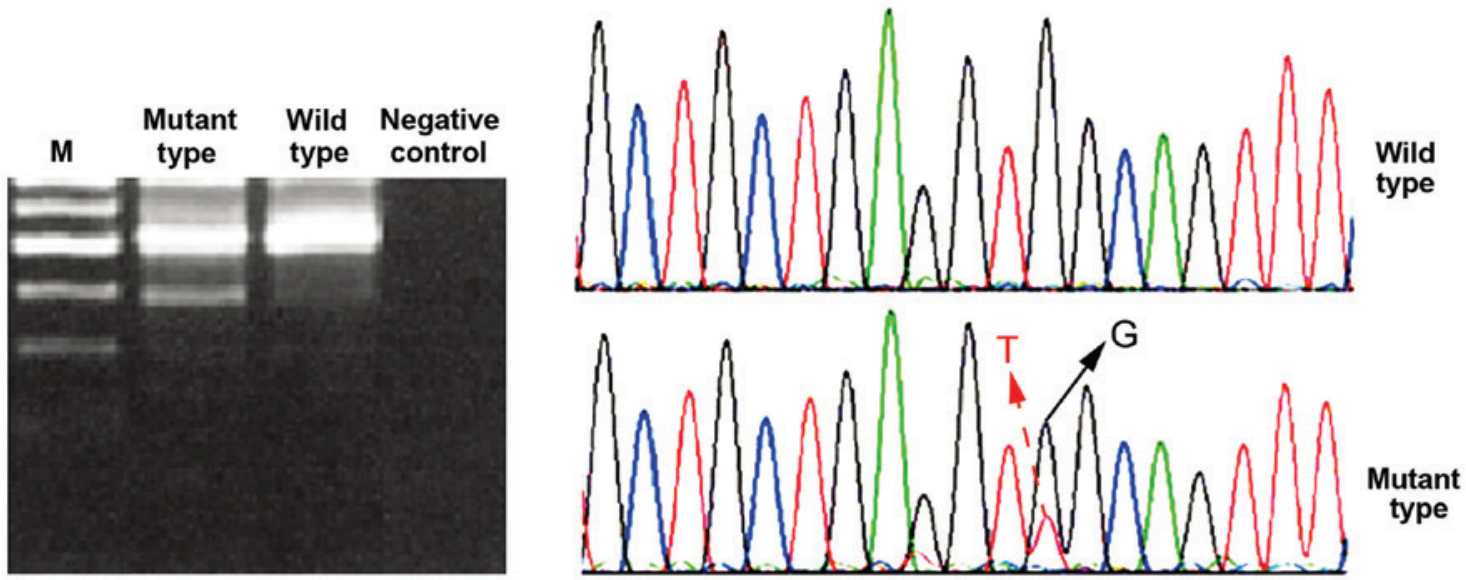

Figure 2. PCR and sequencing results of the MPLW515 mutation (heteroduplex chromatogram; black solid arrow, G peak; red dotted arrow, T peak). $\mathrm{PCR}$, polymerase chain reaction.

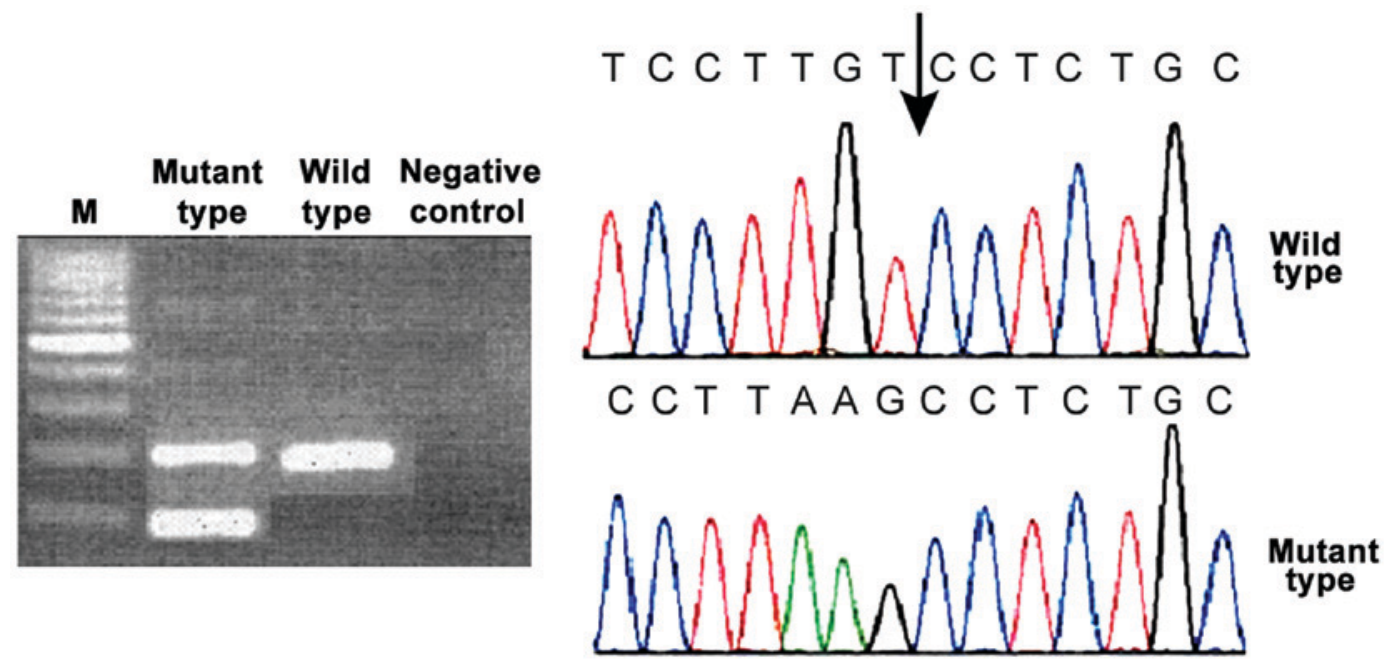

Figure 3. PCR and sequencing results of the CALR mutation (52 bp deletion). PCR, polymerase chain reaction.

wild-type showed only one band. JAK2V617F was located in exon 12. The first base $\mathrm{G}$ was replaced by $\mathrm{T}$ and the codon was changed from V to F. Only V617 codon was affected by the point mutation that causes missense mutation, which did not change the open reading frame. Open reading frame of MPL was not changed either. CALR gene mutations have both 52 bp deletion and 5 bp insertion. All mutations can cause the change of the open reading frame. $\mathrm{C}$ amino acid sequence of CALR protein was changed (Figs. 1-3).

There were $89(57.79 \%)$ cases of JAK2V617F mutations in the ET group, 7 (4.55\%) cases of MPL mutations and $48(31.17 \%)$ cases of CALR mutations. CALR and JAK2V617F mutations at the same time were positive in 7 cases $(4.55 \%)$ and negative in 24 cases $(15.58 \%)$. JAK2V617F positive with 


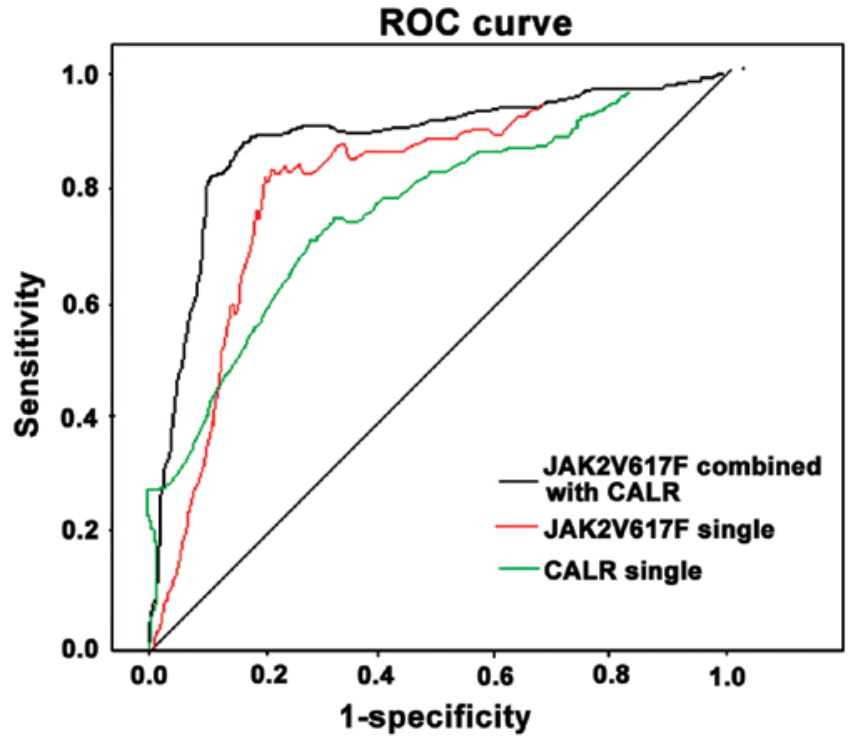

Figure 4. The receiver operating curve (ROC) analysis.

CALR negative were observed in 82 cases (53.25\%), 41 cases (26.62\%) were JAK2V617F negative and CALR positive. In addition, $6(3.19 \%)$ cases were detected with JAK2V617F mutation in the secondary thrombocythemia group, 0 cases of MPL mutation, and 1 case $(0.53 \%)$ of CALR mutation. The positive mutation rate of JAK2V617F and CALR in the ET group was significantly higher than that in the secondary thrombocythemia group, and the difference was statistically significant $\left(\chi^{2}=125.800, \mathrm{p}<0.001 ; \chi^{2}=64.734, \mathrm{p}<0.001\right)$.

ROC analysis. The JAK2V617F average expression level in patients with positive JAK2V617F and CALR mutation at the same time was $0.34 \pm 0.06$. The CALR average expression level was $0.15 \pm 0.04$. Also the average expression levels in patients with negative mutations at the same time were $0.07 \pm 0.01$ and $0.03 \pm 0.01$, respectively. The average expression levels in patients with positive JAK2V617F mutation and negative CALR mutation were $0.58 \pm 0.04$ and $0.04 \pm 0.01,0.08 \pm 0.02$ and $0.22 \pm 0.03$ in patients with negative JAK2V617F mutation and positive CALR mutation.

When JAK2V617F mutation positive was the only diagnostic criteria, the area under the curve (AUC) was 0.721 , $95 \%$ confidence interval (CI) of $0.356-0.925$, sensitivity of $72.4 \%$, specificity of $79.5 \%$ and the cut-off of 0.25 . When CALR mutation positive was the only diagnostic criteria, the AUC was $0.664,95 \%$ CI of $0.291-0.848$ with $68.4 \%$ sensitivity, $82.4 \%$ specificity. The cut-off was 0.09 . When the combined positive CALR and JAK2V617F mutation was used for diagnosis, AUC was $0.862 .95 \%$ CI was $0.467-0.963$. The sensitivity was $85.9 \%$. The specificity was $87.8 \%$ and the cut-off was 0.21 and 0.07 , respectively (Fig. 4).

\section{Discussion}

JAK is a class of non-receptor tyrosine protein kinase. The gene encoding JAK2 is on chromosome 9 (9p24) and it is expressed in almost all tissues. The most obvious structure of JAK is characterized in that the $\mathrm{C}$ terminal has two sections of catalytic and $\mathrm{N}$ terminal has three conserved structural sections. The middle part has two sections. Downstream of JAK signal is protein sub-family of signal transduction and transcriptional activation (STAT). JAK can bind with the conserved BOX1 and BOX2 of cytokine receptor and identify the motif in the cytokine receptor juxtamembrane region. It occurs in a series of phosphorylation under the stimulation of receptors and ligands and selective activate downstream substrates STAT, which translocates to the nucleus and binds with the nuclear specific DNA regulatory elements so as to guide transcription, which is the JAK-STAT pathway (8). The identification of JAK2V617F provides an important clue to explore the pathogenesis of ET.

MPL is thrombopoietin receptor and its peptide sequence has 633 amino acids. Two types of somatic cell mutations MPLW515L/K were confirmed to exist in 5 and $1 \%$ of patients with negative JAK2V617F bone marrow fibrosis (9). MPLW515 was located in the only concurrent region of MPL, which can inhibit the MPL gene spontaneous activation. MPLW515L mutation is located in the suppressed sequence of cytoplasmic and transmembrane region of MPL gene sequence of KWQFP. The mutations within this region can damage the inhibitory function of KWQFP sequence and thus constitutively activate the JAK-STAT signal transduction pathway (10). CALR is $\mathrm{Ca}^{2+}$ binding protein complex, mainly located in the endoplasmic reticulum lumen and the mediated $\mathrm{Ca}^{2+}$ homeostasis can regulate a variety of cellular functions, including the differentiation and maturation of megakaryocytes and platelet formation, integrin mediated signal transduction, immune response, cell apoptosis, proliferation and phagocytosis, wound healing and fibrosis (11). Kampfl et al (12) and Nangalia et al (13) detected CALR exon 9 mutation in most of the JAK2 and MPL negative samples using sequencing method, while CALR gene mutation was not found in a healthy population, lymphatic system tumor, acute leukemia or solid tumors.

We concluded through the study that JAK2V617F and MPL mutations do not cause frameshift, and CALR mutations can lead to the change of open reading. The JAK2V617F and CALR gene mutation rates in the ET group were significantly higher than those in the group of secondary thrombocythemia and the positive MPL mutation rate was only $4.55 \%$. MPL lower positive mutation rate may be related to racial factors (14) and sample size. The innovation of the study is that the three kinds of genes in MPN had certain positive mutation rates based on a previous study (15). Gene analysis for outpatient service with simple increased platelets indicates that the CALR and JAK2V617F mutation can be a specific marker of ET screening. The shortcoming of the study was that it did not involve other types of MPN, which cannot compare the CALR and JAK2V617F mutation in different types of MPN. In addition, the study detected the relative expression levels of JAK2V617F and CALR through quantitative analysis and set positive JAK2V617F, CALR mutation alone and combination as diagnostic criteria respectively, showed that combination use of those two can improve obviously the diagnostic sensitivity, specificity and accuracy. It provides an important reference basis for early clinical recognition.

In conclusion, with the increased JAK2V617F- and CALR-positive mutation rate, the combination of both can 
significantly improve the sensitivity, specificity and accuracy of the diagnosis of ET.

\section{Acknowledgements}

This study was supported by the National Natural Science Fund (81472651/H1604).

\section{References}

1. Barbui T, Thiele J, Vannucchi AM and Tefferi A: Rationale for revision and proposed changes of the WHO diagnostic criteria for polycythemia vera, essential thrombocythemia and primary myelofibrosis. Blood Cancer J 5: e337, 2015.

2. Haider M, Gangat N, Lasho T, Abou Hussein AK, Elala YC, Hanson $\mathrm{C}$ and Tefferi A: Validation of the revised international prognostic score of thrombosis for essential thrombocythemia (IPSET-thrombosis) in 585 Mayo clinic patients. Am J Hematol 91: 390-394, 2016

3. Liew EL, Araki M, Hironaka Y, Mori S, Tan TZ, Morishita S, Edahiro Y, Ohsaka A and Komatsu N: Identification of AIM2 as a downstream target of JAK2V617F. Exp Hematol Oncol 5: 2, 2016.

4. Mahjoub S, Baccouche H, Sahnoun M, Kaabi H, Manai Z, Slama $\mathrm{H}$ and Ben Romdhane N: The JAK 2 mutation in myeloproliferative disorders: A predictive factor of thrombosis. Tunis Med 93: 474-477, 2015 (In French).

5. Okabe M, Yamaguchi H, Usuki K, Kobayashi Y, Kawata E, Kuroda J, Kimura S, Tajika K, Gomi S, Arima N, et al: Clinical features of Japanese polycythemia vera and essential thrombocythemia patients harboring CALR, JAK2V617F, JAK2Ex12del, and MPLW515L/K mutations. Leuk Res 40: 68-76, 2016.

6. Marty C, Pecquet C, Nivarthi H, Elkhoury M, Chachoua I, Tulliez M, Villeval JL, Raslova H, Kralovics R, Constantinescu SN, et al: Calreticulin mutants in mice induce an MPL-dependent thrombocytosis with frequent progression to myelofibrosis. Blood 127: 1317-1324, 2016.

7. Tefferi A, Thiele J and Vardiman JW: The 2008 World Health Organization classification system for myeloproliferative neoplasms: Order out of chaos. Cancer 115: 3842-3847, 2009.
8. Čokić VP, Mitrović-Ajtić O, Beleslin-Čokić BB, Marković D, Buač M, Diklić M, Kraguljac-Kurtović N, Damjanović S, Milenković P, Gotić M, et al: Proinflammatory cytokine IL-6 and JAK-STAT signaling pathway in myeloproliferative neoplasms. Mediators Inflamm 2015: 453020, 2015.

9. Cabagnols X, Favale F, Pasquier F, Messaoudi K, Defour JP, Ianotto JC, Marzac C, Le Couédic JP, Droin N, Chachoua I, et al: Presence of atypical thrombopoietin receptor (MPL) mutations in triple-negative essential thrombocy themia patients. Blood 127: 333-342, 2016.

10. Milosevic Feenstra JD, Nivarthi H, Gisslinger H, Leroy E, Rumi E, Chachoua I, Bagienski K, Kubesova B, Pietra D, Gisslinger B, et al: Whole-exome sequencing identifies novel MPL and JAK2 mutations in triple-negative myeloproliferative neoplasms. Blood 127: 325-332, 2016.

11. Verger E, Cassinat B, Chauveau A, Dosquet C, Giraudier S, Schlageter MH, Ianotto JC, Yassin MA, Al-Dewik N, Carillo S, et al: Clinical and molecular response to interferon- $\alpha$ therapy in essential thrombocythemia patients with CALR mutations. Blood 126: 2585-2591, 2015.

12. Klampfl T, Gisslinger H, Harutyunyan AS, Nivarthi H, Rumi E, Milosevic JD, Them NC, Berg T, Gisslinger B, Pietra D, et al: Somatic mutations of calreticulin in myeloproliferative neoplasms. N Engl J Med 369: 2379-2390, 2013.

13. Nangalia J, Massie CE, Baxter EJ, Nice FL, Gundem G, Wedge DC, Avezov E, Li J, Kollmann K, Kent DG, et al: Somatic CALR mutations in myeloproliferative neoplasms with nonmutated JAK2. N Engl J Med 369: 2391-2405, 2013.

14. Kim SY, Im K, Park SN, Kwon J, Kim JA and Lee DS: CALR, JAK2, and MPL mutation profiles in patients with four different subtypes of myeloproliferative neoplasms: primary myelofibrosis, essential thrombocythemia, polycythemia vera, and myeloproliferative neoplasm, unclassifiable. Am J Clin Pathol 143: 635-644, 2015.

15. Rumi E, Pietra D, Ferretti V, Klampfl T, Harutyunyan AS, Milosevic JD, Them NC, Berg T, Elena C, Casetti IC, et al; Associazione Italiana per la Ricerca sul Cancro Gruppo Italiano Malattie Mieloproliferative Investigators: JAK2 or CALR mutation status defines subtypes of essential thrombocythemia with substantially different clinical course and outcomes. Blood 123: 1544-1551, 2014. 